Int. J. Electrochem. Sci., 11 (2016) $7519-7526$

\title{
ZIF-9 with Enhanced Surpercapacitor and Electrocatalytic for Oxygen Evolution Reaction Performances in Alkaline Electrolyte
}

\author{
Daojun Zhang ${ }^{1}$, Jingchao Zhang ${ }^{1, *}$,Helong Bai ${ }^{2}$, Renchun Zhang ${ }^{1}$, Huaizhong Shi ${ }^{1}$, Baiqing Yuan ${ }^{1, *}$ \\ ${ }^{1}$ Henan Province Key Laboratory of New Optoelectronic Functional Materials, College of Chemistry \\ and Chemical Engineering, Anyang Normal University, Anyang 455002 \\ ${ }^{2}$ College of Chemistry, Jilin University, Changchun 130012 \\ *E-mail: baiqingyuan1981@126.com, zjc19830618@126.com
}

doi: $10.20964 / 2016.09 .01$

Received: 12 May 2016 / Accepted: 7 July 2016 / Published: 7 August 2016

Zeolitic imidazolate frameworks (ZIFs) are a new class of metal-organic materials built by tetrahedral metal ions and different imidazolate ligands. Own to the porous structure, exceptional thermal and chemical stability, ZIFs is considered to be promising candidates in various fields. In alkaline condition, the good catalytic activities of ZIF-9 for oxygen production were confirmed by its low overpotential, small tafel slope and high durability during oxygen evolution reaction. Furthermore, the cycling performance test of ZIF-9 shows that it can afford the highest capacitance after 3150 repeating cycles, then decreased slightly with furthermore charge-discharge cycles. The final specific capacitance of the ZIF-9 is $94.3 \%$ of its maximum value after 6000 cycles. These results show that ZIF-9 might be a capable electrode material for supercapacitor and water splitting electrocatalyst in the future.

Keywords: ZIF-9; Oxygen evolution reactions; Metal-organic frameworks; Supercapacitors; Electrocatalysts

\section{$\underline{\text { FULL TEXT }}$}

(C) 2016 The Authors. Published by ESG (www.electrochemsci.org). This article is an open access article distributed under the terms and conditions of the Creative Commons Attribution license (http://creativecommons.org/licenses/by/4.0/). 